\title{
Analisi dell'applicabilitá della solidarietá contrattuale in Ecuador
}

Analysis of the viability of applying the Contractual Solidarity in Ecuador

\section{Felipe Ignacio Zurita Burbano}

Ricercatore giuridico indipendente

Cittá: Quito

Paese: Ecuador

\section{Francesca Benatti}

Professoressa ass. di diritto comparato Università degli Studi di Padova Cittá: Milano

Paese: Italia

Articolo originale (ricerca)

RFJ, No. 8, Vol I, 2020, pp. 193-219, ISSN 2588-0837

SOMMARIO: Al giorno d'oggi, l'inadempimento degli obblighi contrattuali ha causato un grande dibattito in dottrina, a causa della necessità di relazioni contrattuali meno suscettibili di estinzione e di figure che evitino tale estinzione. Per questo motivo, una parte della dottrina ha preferito optare per rimedi meno ortodossi che consentano di risolvere le controversie senza avviare processi giudiziari. L' articolo analizzerà la fattibilità dell'applicazione di una figura come la solidarietà contrattuale in Ecuador. Inoltre, le caratteristiche del sistema giuridico ecuadoriano consentirebbero l'applicazione di questi rimedi pur con difficoltà attraverso la buona fede. A tal fine, verrà prima analizzato il contratto e il suo inadempimento nell'ambito del diritto civile e nel contesto ecuadoriano. Successivamente, verrà esplorata brevemente la solidarietà contrattuale e l'ultima sezione studierà la possibilità di applicare questa figura nel sistema giuridico ecuadoriano e le conseguenze che ciò comporterebbe. 
PAROLE CHIAVE: diritto dei contratti, responsabilità legale, teoria legale, sistemi legali, dottrina.

ABSTRACT: Nowadays, the breach of contractual obligations has caused great debate in the law academy, this due to the need for contractual relationships that are less susceptible to extinction and figures that avoid this extinction. For this reason, a part of doctrine has preferred to opt for less orthodox remedies that allow the resolution of controversies without initiating judicial processes. Due to the above, this article will analyze the feasibility of applying a figure such as contractual solidarity in Ecuador. Furthermore, the characteristics of the Ecuadorian legal system would allow the application of these remedies despite difficulties through good faith. For this purpose, the contract will first be analyzed as a figure and the breach within the field of Civil law and in the Ecuadorian context. Afterwards, contractual solidarity will be explored briefly, and the last section will study the possibility of applying this figure in the Ecuadorian legal system and the consequences that this would entail.

KEY WORDS: contract law, legal liability, legal theory, legal systems, doctrine.

\section{INTRODUZIONE}

Gli ordinamenti di civil law regolano con modalità differenti la risoluzione contrattuale per inadempimento. Al modello giudiziale si è oggi affiancato in alcuni sistemi quello extragiudiziale unilaterale, che consente al creditore di risolvere il contratto nei casi di inadempimento della prestazione da parte del debitore senza ricorrere al giudice. L'art. 1226 del codice civile francese prevede che "il creditore può, a proprio rischio, risolvere il contratto mediante notifica. Tranne che in caso di emergenza, deve prima dare un preavviso formale al debitore inadempiente per rispettare il proprio impegno entro un termine ragionevole". Parte della dottrina (Dellacasa,2015) ha sottolineato come tale figura sembri consentire una maggiore rapidità e minori costi, adattandosi alle esigenze del mondo degli affari. 
Tuttavia, il tema della risoluzione per inadempimento appare assumere oggi, in America Latina, profili delicati a causa delle misure restrittive adottate dai governi per salvaguardare la salute pubblica di fronte alla pandemia di Covid19: la maggior parte dei contratti non è stata adempiuta o non potrà esserlo, le persone non sono nelle condizioni di pagare i debiti $\mathrm{o}$, addirittura, preferiscono non farlo e risparmiare quanto possibile al fine di avere i mezzi necessari per affrontare un possibile contagio. Inoltre le risorse economiche devono essere riorientate per evitare un collasso nel settore della sanità pubblica. Tale situazione complessa si rinviene in Ecuador, nonostante le restrizioni siano state progressivamente diminuite: il lavoro si è fermato, le aziende, non producendo, non hanno una sufficiente liquidità per garantire posti di lavoro e nuovi investimenti, Si tratta di una economia quasi bloccata, anche per il terrore di nuove ondate di contagi.

Mentre si dubita dell'efficacia delle nozioni tradizionali di “impossibilità sopravvenuta” ed "eccessiva onerosità" per fronteggiare la pandemia, la possibilità di utilizzare la solidarietà per imporre un obbligo di rinegoziazione o modificare i contratti tutelando parti inadempienti a causa della situazione difficile è una delle soluzioni avanzate sia in America Latina sia in Europa. In Italia, infatti, anche la dottrina che "ha spesso denunziato l'uso, a tratti spregiudicato, dell'argomento costituzionale nel ragionamento civilistico, apparsogli, in più occasioni, un comodo espediente per sottrarsi alla fatica del concetto e per veicolare, tramite ragionamenti breviloqui, soluzioni che avrebbero dovuto argomentarsi attraverso puntuali norme positive, agevolandone così il controllo razionale. Tuttavia, nei tempi eccezionali che viviamo, le norme positive (tese, come sono, alla distruzione del vincolo più che alla sua conservazione) certamente non soccorrono. Proprio per questo, oggi, si possono e si devono chiamare in campo la buona fede contrattuale e $\mathrm{i}$ principi costituzionali, per preservare, loro tramite, l'economia di scambi congegnati in tempi normali ma irrimediabilmente alterati, per cause di forza maggiore, in tempo di emergenza" (Benedetti-Natoli, 2020). 
In questo scenario, deve, dunque, essere valutata l'applicabilità della solidarietà contrattuale e la possibilità di una sua eventuale estensione a tutti i casi nei quali ricorrono gravi squilibri contrattuali. Il problema appare particolarmente avvertito in Ecuador in assenza di quello che pare essere un contesto giuridico, economico e sociale adatto. Come ha rilevato una autorevole dottrina "la parola solidarietà evoca concetti e dati dell'esperienza come amicizia, carità, benevolenza e accostata a diritto suggerisce connessioni, distinzioni, contrapposizioni tra quelli ed il fenomeno giuridico ...si agita...tutta una sostanza di atteggiamenti spirituali e relazioni con il prossimo, quello al quale si è legati da particolari rapporti e quello invece anonimo con il quale entriamo in contatto in maniera casuale, ancorché assidua" (Rescigno, 2006 pp. 6-8.) La figura della solidarietà contrattuale, nata come contrappeso alla totale libertà delle parti che caratterizzava la visione tradizionale del contratto, si riviene oggi in numerosi ordinamenti tra i quali quello francese e italiano.

\section{IL CONTRATTO, L' INADEMPIMENTO E L'ORDINE LEGALE ECUADORIANO}

La base dello scambio economico di tutte le società è il contratto che nasce dalla libera volontà delle parti contraenti: "la libertà contrattuale si riferisce a quella libertà che il sistema giuridico garantisce ai cittadini di stipulare accordi che in un determinato quadro giuridico possono essere validi e legalmente applicabili" (Chamié, 2018, p.195) Nel contesto ecuadoriano, il codice civile lo definisce nel modo seguente: "il contratto o la convenzione è un atto con il quale una parte concorda con un'altra di dare, fare o non fare qualcosa. Ogni parte può essere costituita da una o più persone". (Codice civile, 2005, art. 1454). L' accordo ha forza di legge tra le parti, che possono determinare liberamente i benefici e i rispettivi obblighi. Se in un contesto ideale ogni contraente ricava un vantaggio, nella realtà ciò non sempre avviene. Bisogna distinguere, perciò, i casi in cui questo succede per un normale rischio d'impresa da quelli nei quali la perdita deriva da abusi o scorrettezze, magari aggravate da asimmetrie. 
In tal senso Momberg ha rilevato come "in termini generali, la forza vincolante del contratto implica che le parti devono rispettare gli obblighi che hanno validamente contratto, avendo questo forza vincolante per loro e quindi esecutività. Nella sua concezione tradizionale presuppone anche l'intangibilità del contratto, in modo che né le parti (con l'ovvia eccezione del mutuo consenso) né il giudice possano variare i termini di un contratto validamente concluso". (citato in Accatino, 2015, p.37).

L'obiettivo del rapporto contrattuale, infatti, è che duri e gli obblighi contrattuali determinati siano effettivamente adempiuti e quindi soddisfino gli interessi di entrambe le parti.

Tuttavia, "gli effetti delle obbligazioni, che compongono le disposizioni delle parti contraenti in tutti i contratti, sia consensuali o meno, ruotano, come già accennato, attorno a due possibilità: esecuzione o inadempimento. È sia l'inadempimento da parte dell'uno o dell'altro contraente, sia la sua esecuzione che sono previsti dalle parti. Ciò è evidente quando esse contemplano espressamente la violazione delle obbligazioni e ne stabiliscono la sanzione"(Lecuyer,2010, p.47).

Nella sua essenza, la teoria tradizionale configura il contratto come una relazione di reciprochi obblighi delle parti diretti a soddisfare reciproci interessi per cui l'inadempimento di un contraente si traduce in una grave insoddisfazione dell'altro. Pertanto,

... l'obbligazione è concepito come un dovere di collaborazione intersoggettiva, la cui funzione è proprio quella di soddisfare l'interesse del creditore, soddisfazione per la quale il debitore deve impiegare tutti i suoi sforzi, alle condizioni corrispondenti alla natura della prestazione, alle disposizioni generali e specifiche di legge, alle obbligazioni derivanti dall'esercizio dell'autonomia privata, oltre alle clausole contrattuali. La soddisfazione del creditore, generalmente effettuata dal debitore, l'unico ad esso obbligato, ha l'effetto di estinguere l'obbligazione, poiché la sua funzione è stata 
svolta e, di conseguenza, rilascia il debitore (solutio). L'insoddisfazione del creditore, totale o parziale, transitoria o definitiva, implica un'anomalia, che di solito è attribuita al debitore e, più specificamente, al suo comportamento scorretto, indipendentemente dall'onere della prova"(Hinestrosa, 2016, p. 5).

Nei casi di inadempimento il codice civile ecuadoriano stabilisce il diritto del creditore di chiedere l'adempimento: “ ogni obbligazione personale dà al creditore il diritto di eseguirla su tutti i beni immobili o mobili del debitore, siano essi presente o futuri (C.C, 2005, art. 2387)". Ciò implica che il giudice può obbligare il debitore all'adempimento. Non si tratta, però, di "una creazione artificiale, qualcosa costruito senza fondamento nella vita reale. Al contrario, l'obbligazione del contratto trova solide basi nei vari settori rispetto alla libertà creativa dell'uomo; all'iniziativa privata, come generatrice di ricchezza e di conseguenza attivatrice del benessere sociale ..." (GarcíaAgúndez, 1983, p.7).

Benché normalmente le parti non violano i doveri nascenti dalla lex contractus liberamente voluti, può verificarsi un inadempimento totale o parziale, cioè una deviazione rispetto al programma contrattuale pattuito (Gómez, 2007 p.7), il che fa sorgere in capo al creditore il diritto a ricevere ciò che è dovuto più i danni causati. Secondo il codice civile ecuadoriano il risarcimento dei danni comprende danni consequenziali e perdita di profitti, indipendentemente dal fatto che derivino dal mancato rispetto dell'obbligo o dall'adempimento imperfetto o dal ritardato rispetto (CC, 2005, art. 1572). Tuttavia, è riconosciuto che eventi di forza maggiore o caso fortuito che non erano prevedibili al momento di conclusione del contratto e indipendenti dalla sfera di controllo del creditore possono giustificare l'inadempimento. E' infatti una "applicazione della massima secondo la quale" nessuno è obbligato all'impossibile" "(Castro Ruiz, 2015, p.442).

In alcuni ordinamenti, si ha una esemplificazione legislativa delle ipotesi che possono essere ricomprese nel caso fortuito o nella forza maggiore; in altri vi è una definizione generale. Un esempio del primo modello è l'Ecuador, in cui il 
codice civile stabilisce che "si chiama forza maggiore o evento fortuito, l'imprevisto a cui non è possibile resistere, come un naufragio, un terremoto, l'arresto di nemici, gli atti di autorità esercitati da un pubblico ufficiale, ecc." (C.C, 2005, Art.30). Muovendo da questa previsione normativa, la dottrina e la giurisprudenza si sono concentrate sulla individuazione di altre fattispecie analoghe. Nel contratto di lavoro, ad esempio, esse ricorrono quando il fatto che provoca il licenziamento è imprevisto e irrimediabile per il datore di lavoro, e il suo verificarsi non è imputabile al datore di lavoro se esiste una relazione di causa ed effetto tra l'evento fortuito o la forza maggiore e la fine del rapporto. (Castro, 2015, p.15)

L'ordinamento italiano prevede all'art. 1218 c.c. it. che il debitore che non esegue esattamente la prestazione dovuta è tenuto al risarcimento del danno se non prova che l'inadempimento o il ritardo è stato determinato da impossibilità della prestazione derivante da causa a lui non imputabile In senso analogo l'art. 1256 c.c. afferma che l'obbligazione si estingue quando, per una causa non imputabile al debitore, la prestazione diventa impossibile. E' stato messo in luce, tuttavia, che l'"impossibilità non costituisce una nozione astratta, valida per tutti i rapporti di obbligazione, e non esprime un carattere dell'evento, ma è un giudizio che si formula col riferirsi alla prestazione dedotta in obbligazione" (Gambino, 2015, p 192) con riferimento a ciò che le parti hanno espressamente disposto o implicitamente convenuto e, quando, occorrono integrazioni o specificazioni ulteriori in base ad un criterio generale di ragionevolezza ed efficienza" (Trimarchi, 2008, p. 349), secondo la natura e gli scopi del contratto.

Sia in Ecuador che in Italia sembra invocabile l'impossibilità sopravvenuta della prestazione per factum principis nel caso del Covid-19 in relazione alle misure governamentali con le quali è stata interrotta o limitata l'attività produttiva. Tuttavia, posto che essa non riesce a disciplinare tutte le fattispecie verificatesi in concreto, nella dottrina ecuadoriana si assiste ad un dibattito sulla opportunità di estendere l'uso della buona fede in modo da fronteggiare in modo più flessibile ed adeguato i problemi causati dalla pandemia e rendere più efficienti le relazioni contrattuali. Una soluzione 
potrebbe essere il ricorso alla figura della inesigibilità: qualora la prestazione fosse ancora possibile, ma comportasse un sacrificio eccezionale e irragionevole, l'impossibilità si potrebbe qualificare come "inesigibilità" mediante l'applicazione del principio di buona fede nel rapporto obbligatorio e nel contratto. Si consideri un'impresa che non adempie perché i suoi fornitori sono in quarantena e non riesce se non con sforzi abnormi e troppo onerosi. Luigi Mengoni avverte come l'inesigibilità "non appartenga agli impedimenti oggettivi della prestazione...(e) può determinarsi in ragione non solo di preminenti interessi non patrimoniali inerenti alla persona del debitore, ma anche di un pericolo imprevedibile di danno grave ai suoi beni, in particolare alla produttività della sua azienda" : la buona fede impone, infatti, un limite all'esercizio del diritto di credito.

\section{LA SOLIDARIETA'CONTRATTUALE}

Dall'introduzione del Code civil del 1804, la dottrina si è concentrata sull'esame della natura e dei limiti del principio dell'"autonomia privata", criticato da alcuni autori per essere un "il concetto illimitato di libertà che deriva dall'instaurazione di obblighi con il debitore. La libertà di contrattare mette in secondo piano l'intervento statale, muovendo dal volontarismo esacerbato e dal liberalismo economico" (Tito, 2019). Da questa visione origina la teoria della solidarietà che cerca di evitare l'abuso del concetto di libertà contrattuale e anticipa l'inizio delle idee sociali dell'intervento statale attivo nelle relazioni economiche:

Infatti "a causa delle incoerenze generate dalle espressioni puramente formali del principio dell'autonomia della volontà, la dottrina del "Solidarismo contrattuale, o del" Diritto sociale "è sorta in Francia a metà del XIX secolo, e afferma che ognuna delle parti coinvolte in un contratto debba tenere conto dell'interesse del suo contraente, al fine di garantire che convergano efficacemente nel rapporto contrattuale i classici postulati ius-privatistici, come la sicurezza giuridica e la stabilità con altri principi quali la proporzionalità, la coerenza, la lealtà, la giustizia e soprattutto la solidarietà ". (Morgestein, 2015, p.196) 
È così che viene sviluppata un`altra concezione secondo la quale i contraenti mantengono ancora la libertà di determinare ciò che è conveniente per loro, ma allo stesso tempo considerano da una prospettiva sociale e solidale ciò di cui l'altra parte ha bisogno e non solo il loro interesse personale. Ciò tenderebbe a garantire il principio di giusto equilibrio e stabilità contrattuale.

Questo comporta l'affidamento in "un atteggiamento di collaborazione, di mutuo aiuto, indipendentemente da quanto distanti possano essere i loro interessi e porta a respingere gli atteggiamenti contraddittori che le parti possono avere, in quanto si basa precisamente su principi quali buona fede, lealtà e coerenza contrattuale, tra gli altri. I solidaristi criticano il diritto classico perché connotato da uno scopo egoistico e ritengono che la legge e i giudici debbano intervenire per evitare abusi e ristabilire l'equilibrio nei contratti" (Bernal, 2007, p.18).

Considerando la solidarietà come un meccanismo che reincorpora l'equilibrio sociale, così da impedire il compimento di condotte sleali e strettamente egoistiche, viene limitata la rigidità del diritto contrattuale. Tuttavia, essa non potrebbe esistere senza il principio di buona fede, che si traduce in fiducia e lealtà tra le parti: "la solidarietà contrattuale si basa sul principio di buona fede, che funge da parametro di etica contrattuale. La buona fede modella l'autonomia privata perché determina la forza dell'obbligo e (impone) che i contraenti debbano comportarsi con lealtà e onestà. La solidarietà entra e assume rilevanza nei contratti attraverso la clausola generale di buona fede". (Benítez, 2013, p.147).

Nell'ordinamento italiano, tale clausola generale è divenuta una "sorta di valvola di sicurezza che allarga le maglie del tessuto normativo, lo salda alla storia e ai contegni mutevoli degli uomini e agevola gli scopi di chi nel diritto scorge un organismo aperto e pronto a espandersi sulla base delle istanze che la società accumula nel corso del tempo" (Corradini,1970, p. 491). Essa ha subito una progressiva evoluzione: mentre all'inizio era usata in modo contenuto considerando l'interesse dell'operazione economica e quindi in connessione alle lacune delle norme e a quelle che sorgevano necessariamente dalla lettura del contratto, è stata allargata progressivamente portando 
alla sua concreta applicazione attraverso la costruzione di $\backslash$ figure dogmatiche ormai affermate quali l'abuso del diritto, il venire contra factum propium, i doveri di protezione e ed è usata come fonte d' integrazione superando quella che è l'equità dell'art. 1374 c.c. it.

L'estensione nel suo utilizzo è dovuta anche all'indirizzo confermato dalla Corte Costituzionale che collega la buona fede all'art 2 della Costituzione e ad un mutato clima giuridico/ culturale, che attenua il rigido positivismo per affermare il ruolo creativo della giurisprudenza. Quella italiana ha, infatti, affermato ormai l' "acquisita consapevolezza della intervenuta costituzionalizzazione del canone generale di buona fede oggettiva e correttezza, in ragione del suo porsi in sinergia con il dovere inderogabile di solidarietà di cui all'art. 2 Cost., che a quella clausola generale attribuisce all'un tempo forza normativa e ricchezza di contenuti, inglobanti anche obblighi di protezione della persona e delle cose della controparte, funzionalizzando così il rapporto obbligatorio alla tutela anche dell'interesse del partner negoziale" (Cassazione civile sez. un., 15/11/2007, n. 23726) .

La dottrina ha, però, messo in luce come non si sia mai offerto un criterio di concretizzazione del contenuto solidarista della buona fede, limitandosi ad enunciarlo (Grondona, 2004). Tale aspetto è decisivo perché permetterebbe di estendere il concetto di solidarietà ai casi nei quali è effettivamente necessario e fondato su esigenze di tutela della persona. Spesso, invece, serve solo come argomento retorico, privo di contenuto, per giustificare soluzioni di rottura o imprevedibili, che diversamente sarebbero difficilmente comprensibili o accettabili. Questo ha riflessi negativi perché non aiuta un dibattito obiettivo sul tema che resta pertanto sul piano dell'emozione. Qualora si desideri perseguire una effettiva solidarietà, appare necessario individuare le ipotesi o i criteri di applicazione e i limiti in modo da evitare soluzioni che possano apparire teoricamente corrette ma nella pratica ingiuste oppure non seriamente giustificate. Bisogna evitare, insomma, un solidarismo indifferenziato e riduttivo a favore di una attenzione alle situazioni effettivamente meritevoli.

La valorizzazione della solidarietà attraverso la buona 
fede può consentire in determinate circostanze una migliore rimodulazione degli interessi delle parti:

...la solidarietà contrattuale mette in discussione il dogma dell'autonomia della volontà secondo la quale l'individuo è il miglior conoscitore dei suoi interessi. Mette in dubbio l'idea che le parti dovrebbero essere le uniche a valutare il contratto e (dubita che) il fatto che sia negoziato implichi necessariamente che sia equilibrato e che sia impossibile intervenire al fine di garantire la certezza del diritto. Questa dottrina cerca di conciliare i classici imperativi di stabilità e certezza del diritto con principi come la solidarietà.”. (Bernal, 2007, p.17)

Tale concezione ha, dunque, lo scopo sia di affrontare l'inadempimento contrattuale sia di conformare le modalità con cui le parti cooperano tra loro. Per questo motivo, essa è presente non solo in ogni momento della vita del contratto, ma anche prima della sua stipulazione e successivamente alla sua estinzione.

Nella fase precontrattuale la solidarietà tende a una "trattativa pulita" basata sulla buona fede, che svolge un ruolo fondamentale. Nel diritto italiano l'art. 1337 c.c. it.. impone alle parti di comportarsi secondo buona fede nella fase di formazione del contratto. Il dovere è consacrato ormai in tutti gli ordinamenti e anche nei progetti internazionali ed europei. L'art. 2.1.15 dei Principi Unidroit stabilisce che "(1) Una parte è libera di negoziare e non è responsabile per il mancato raggiungimento di un accordo (2) Tuttavia, una parte che negozia o interrompe le trattative in malafede è responsabile delle perdite causate all'altra parte. (3) È in malafede, in particolare, che una parte cominci o continui le trattative quando non intende raggiungere un accordo". La medesima norma si ritrova all'art. 2: 301 dei PECL. E analogamente all'art. II.-3: 301 del DCFR prevede che "(2) Una persona impegnata nelle trattative ha il dovere di negoziare secondo buona fede e correttezza e di non interromperle violando la buona fede e la correttezza. Questo dovere non può essere escluso o limitato dal contratto. (3) Una persona che lo ha violato è responsabile 
per qualsiasi danno causata all'altra parte dalla violazione. (4) ̇̀ in contrasto con la buona fede e l'equità, in particolare, per una persona avviare o proseguire le trattative senza alcuna reale intenzione di raggiungere un accordo con l'altra parte ".

Durante la relazione contrattuale può servire a valutare i comportamenti delle parti, impedendo determinati abusi: infatti, "oltre ad un semplice obbligo passivo di rispetto, è incluso anche un obbligo attivo di collaborare alla realizzazione dell'oggetto del contratto" (Bernal-Fandiño, 2007, p.24).

Infine, nella fase post-contrattuale, la solidarietà contrattuale tutela gli interessi delle parti, controllando le condotte successive che potrebbero ledere il principio di lealtà e di affidamento. Si pensi, ad esempio, agli obblighi di segreto che ciascuna parte deve mantenere anche dopo la fine del rapporto. Si può fare un'altra ipotesi: durante l'esecuzione di un contratto una parte viene a conoscenza di un affare a seguito della natura del suo incarico e lo conclude dopo la estinzione del contratto. Anche qui nasce l'obbligo per buona fede di darne comunicazione e di fare partecipe dei vantaggi anche il vecchio contraente.

Nel momento attuale sembra rilevante esaminare il ruolo che potrebbe avere la buona fede durante la vigenza del rapporto: soprattutto perché

"è necessario adeguare le prestazioni mediante rinegoziazione o adattamento e cercare il soddisfacimento dellıinteresse contrattuale delle parti prima di frustrarlo completamente e terminare il rapporto. In questo modo, si rinviene il dovere di ripristinare l'equilibrio delle prestazioni secondo uno spirito di cooperazione e solidarietà.". (Chamié, 2008, p.133). Ciò permetterebbe inoltre di mantenere e preservare i principi dell'equilibrio contrattuale rimodulandone il contenuto, nei casi identificati dalla legislazione come "forza maggiore o evento fortuito" alla luce della situazione pandemica e della crisi economica profonda.

Sebbene teoricamente la dottrina della solidarietà contrattuale possa fornire una soluzione a rimedio dei rischi che talvolta discendono dall'autonomia privata $\mathrm{e}$ 
dall'inadempimento contrattuale, è stata fortemente criticata da coloro che aderiscono alla teoria classica. Si è obiettato che la solidarietà è lontana dalla realtà in cui vengono creati i contratti. Inoltre, è stata discussa l'interferenza nell'autonomia privata che è considerata la principale caratteristica di differenziazione con la dottrina tradizionale. A queste critiche si è opposto come, "nonostante le obiezioni dei suoi oppositori, la solidarietà non solo rafforza le figure esistenti, ma fornisce loro una nuova interpretazione e un nuovo uso. Questa scuola ha una visione dinamica dei principi di buona fede ed equità, da cui emergono nuovi doveri "(Bernal, 2007, p.19).

La solidarietà contrattuale può essere definita come una figura distante dalla classica visione del contratto fissata nei codici ottocenteschi, che mira a proteggere i principi di equilibrio, lealtà e cooperazione tra le parti in ciascuna delle fasi contrattuali.

\section{3.-VIABILITÀ DELL'APPLICAZIONE DELLA SOLIDARIETÀ DEL CONTRATTO IN ECUADOR}

\section{a) LA GIURISPRUDENZA COME FONTE DI DIRITTO}

Nonostante in Ecuador la figura della solidarietà fosse estranea alla disciplina del contratto, a partire dal 2008, con la Costituzione di Montecristi, è iniziato un percorso che può essere definito "neo-costituzionalista" e di conseguenza si assiste ad un primo tentativo della giurisprudenza di costituzionalizzazione del diritto privato (Eyzaguirre, 2013, p. 153). Il tema richiama il dibattito sviluppatosi in Germania sulla Drittwirkung, dove la dottrina ha avvertito che "la differenza tra legislazione e giurisdizione, inizialmente concepita soltanto in termini qualitativi, appare sempre più evanescente" (Oeter, 2018, p. 202)

Si determina, dunque, un cambio di paradigma: quando è affermata la necessità di introdurre una figura come la solidarietà contrattuale in un sistema di civil law, sembrerebbe inevitabile ritenere che debba avvenire per legge. Tuttavia, la giurisprudenza francese ha dimostrato il contrario: "la sentenza Expovit, della della Corte di cassazione francese, del 25 febbraio 
1992, è stata interpretata come obbligo di solidarietà del datore di lavoro nei confronti del lavoratore. In questo caso, un dipendente è stato licenziato per cessazione della sua posizione. Giorni dopo, però, ne era stato assunto un altro per una mansione le cui competenze erano simili a quelle del dipendente licenziato giorni prima. La Corte ha ritenuto che il datore di lavoro avrebbe dovuto adempiere il contratto di lavoro in buona fede, assicurando l'adattamento dei dipendenti all'evoluzione del loro lavoro (Bernal-Fandiño, 2007, p.27). Deve essere segnalato, poi, che la riforma del 2016 del diritto contrattuale, recependo numerose soluzioni già precedentemente consacrate dalla giurisprudenza, ha ampliato il ruolo della buona fede dalla fase di esecuzione del contratto a principio direttivo che deve guidare l'opera del giudice, riconoscendo, poi, espressamente il dovere di comportarsi "imperativamente" secondo buona fede nelle trattative (art. 1112). (Bernal-Fandiño, 2007, p.22).

Anche in Colombia è stata la giurisprudenza costituzionale a consentire l'ingresso di principi discendenti dalla solidarietà contrattuale: "la giurisprudenza ha evidenziato che determinati settori, come quello finanziario o dell'assicuratore, operando in settori di interesse pubblico richiedono un maggiore incisione della loro libertà contrattuale; quindi, l'autonomia privata è più limitata rispetto ad altri settori" (Bernal-Fandiño, 2016, p.66).

E' vicina la tendenza italiana. Come sottolineato, infatti, di fronte ad una pluralità di soluzioni ormai il giudice "non segue né la via classica di un contemperamento degli interessi direttamente implicati né quella prospettata durante la stagione ideologica dell'uso alternativo del diritto di seguire la graduatoria designata in partenza secondo una tavola di valori definita alla stregua di parametri esterni, ma utilizza semmai il riferimento ai criteri di solidarietà di cui all'art. 2 cost., come il procedimento idoneo a legittimare la soluzione più corretta, proprio in quanto capace di assorbire la conflittualità degli interessi in un contesto più ampio e generale.. in tal modo la solidarietà tende a diventare un modo d'essere dell'esperienza giuridica" (Lipari, 1997, p. 12). Questo ha portato, però, anche a decisioni controverse, tra le quali si possono ricordare le ordinanze 248/13 e 77/14 che hanno affermato la possibilità 
del giudice di dichiarare nulla ex art. 1418 c.c. la caparra confirmatoria per violazione dell'art. 2 Cost. L'indirizzo, che ha aperto un ampio dibattito in dottrina sulla opportunità di applicazione diretta dei principi costituzionali, non ha trovato un unanime accoglimento in giurisprudenza.

\section{b) SOLIDARIETA' CONTRATTUALE NELLA GIURISPRUDENZA ECUADORIANA}

Nonostante la scarsità di studi recenti in materia, è possibile riscontrare una maggiore apertura giurisprudenziale al riconoscimento della solidarietà contrattuale, invocata attraverso la clausola generale di buona fede prevista nel codice civile.

Già all'inizio della fase neocostituzionalista in Ecuador, può essere riscontrata un'applicazione diffusa della buona fede nelle sentenze, connotata, però, da una adesione testuale alla norma. Ciò è dimostrato dalla sentenza n. 373-2009 della Sala civile e commerciale della Corte nazionale di giustizia del 2009. In questa decisione in presenza di un'erronea interpretazione delle norme corrispondenti ai contratti di acquisto e vendita a condizione, essa ha statuito che:

"non avendo esplicitamente indicato il momento in cui deve essere adempiuto il secondo di questi obblighi, ovvero la celebrazione dell'atto pubblico di vendita, devono essere applicate le regole di interpretazione dei contratti, in particolare le disposizioni dell'articolo 1576 del codice civile che stabilisce che conosciuta l'intenzione delle parti contraenti, bisogna aderire ad essa piuttosto che al tenore letterale, nonché all'art. 1579 di tale codice, che stabilisce che nei casi in cui non appare alcuna volontà, al contrario, bisogna applicare la regola di interpretazione più adatta alla natura del contratto; e l' art. 1562 ibidem, norma che determina che i contratti devono essere eseguiti in buona fede, e quindi obbligano, non solo a ciò che è espresso in essi, ma a tutto ciò che deriva dalla natura dell'obbligazione, o che, per legge o costume, ne fa parte" [...] (Corte 
Nacional de Justicia, Sala de lo civil y Mercantil, R.03732009, f.7)

Analogamente nella sentenza n. 0228-2012, relativa ad un caso di inadempimento contrattuale di una compagnia assicurativa, si sottolinea il ruolo della buona fede, pur senza un'analisi approfondita. La Corte stabilisce quanto segue:

[...]è anche un contratto, in cui la buona fede occupa un posto fondamentale, proprio perché l'assicurato non ha fini di lucro e perché (la buona fede) si manifesta dalla fase precontrattuale e ovviamente durante la sua esecuzione. In questo contesto, il modo in cui l'assicurato può far valere i propri diritti, reclamare e ricevere il risarcimento concordato, nel caso in cui l'evento o il reclamo si sia verificato, come la Legge stessa lo chiama, deve essere esaminato in modo speciale" (Corte Nacional de Justicia Sala de lo Civil y Mercantil, No.0228-2012, f.9).

In questa evoluzione appare rilevante il caso deciso dalla Sala de los Civil y Mercantil de la Corte Nacional de Justicia nel 2014 ai sensi della risoluzione n. 0199-2014. La società Prophar aveva ricorso contro la sentenza della Prima Camera civile di Pichincha per avere, senza motivazione, ridotto l'importo del risarcimento al quale era stata condannata Merck Sharp. Questo integrava un'inadeguata applicazione degli articoli in materia di danni e pregiudizi nel codice civile ecuadoriano, posto che Merck Sharp si era comportata durante le trattative con Prophar in malafede. Nell'analizzare il caso, la Corte Nacional fonda la sua motivazione sul dovere di buona fede nella fase precontrattuale attraverso anche un'analisi comparatistica:

"nella fase precontrattuale, nella tradizione dell'Europa continentale, è imposto un dovere di condotta adeguato ai parametri stabiliti dalla buona fede, che è generalmente intesa come dovere di lealtà e cooperazione...Il riconoscimento del principio di buona fede e lealtà commerciale, non espressamente come regola di interpretazione del testo dei Principi, ma come standard obbligatorio di comportamento per 
le parti contraenti, è una delle innovazioni più grandi e di maggior successo presentate dai Principi rispetto ad altri testi di diritto uniforme [..] (Corte Nacional de Justicia, Sala de lo Civil y Mercantil, R.0199-2014, fj.46).

La Corte Nacional stabilisce, dunque, che la buona fede, in quanto principio fondamentale del contratto, deve essere presente nella fase precontrattuale e condanna la società che ha violato tale dovere. Questo caso, a differenza dei primi due, analizza la buona fede come principio che avvolge in tutto il contratto e si discosta dal metodo tradizionale adottato dalla maggior parte dei giudici ecuadoriani in materia in buona fede e solidarietà. Tuttavia esso è ancora ampiamente utilizzato e non consente una comprensione e concretizzazione efficace di queste figure a differenza di quanto avviene in Francia o in Italia. Ciò non dovrebbe significare un abbandono del metodo testualista, ma la capacità di moderarlo alla luce della situazione concreta e delle aspettative ragionevoli delle parti: la buona fede, infatti, serve a individuare quei doveri strettamente connessi al contratto e impone di evitare comportamenti volti a ledere l'altro contraente, a causare sorprese inaspettate, a minare il senso dell'operazione economica, a sfuggire a impegni presi.

Nonostante le sentenze della Corte Nacional mostrino l'uso da parte dei giudici della buona fede contrattuale nella risoluzione delle controversie, esse non costituiscono precedenti vincolanti e questo rende difficile una sua compiuta affermazione. Possono, però, costituire il fondamento per un successiva introduzione consapevole della solidarietà.

Tuttavia si nota come proprio "nei sistemi che non conoscono il vincolo dei precedenti...il decidere per valori sta come solitaria esperienza di un giudice o di una corte" (Irti, 2020, p. 145) e determina il rischio di un irrazionalismo e imprevedibilità delle decisioni. La buona fede si colloca in una ambiguità da cui "possono derivare esiti esattamente contrapposti...una sorta di orgia giurisdizionale ovvero una cautela dei giudici che può rivelarsi eccessiva in relazione al momento storico. Significativamente, le fonti romane proclamano che bona fides quae in contractibus exigitur aequitatem summam desiderat. E' 
l'equilibrio nel decidere nella sua dimensione più intensa quello che la buona fede esige" (Castronovo, 1987, p. 29)

\section{C) L'ECCEZIONE DI BUONA FEDE}

La solidarietà contrattuale è espressione della buona fede e si concretizza nella fiducia (tutelata) che avrà luogo una trattativa "leale", che il contratto sarà eseguito e, in tal caso, il risultato finale sarà a vantaggio di entrambe le parti. Tuttavia questo non sempre avviene. In Ecuador, ma non solo, a volte l'idea del guadagno personale prevale su qualsiasi altra considerazione. Qualora il creditore possa ricavare dei vantaggi rispetto al debitore, potrebbe essere tentato a comportarsi in modo opportunistico o abusivo così da soddisfare unicamente i suoi interessi. La solidarietà tende, invece, a proibire "qualsiasi abuso che la controparte possa compiere di tale situazione (abuso del diritto, frode, uso) per ottenere un vantaggio ingiusto o iniquo (arricchimento senza causa o con una causa illegale basata sullo sfruttamento), generando una evidente sproporzione tra le prestazioni (squilibrio contrattuale, mancanza di equivalenza). Si rompe l'equivalenza tra le posizioni giuridiche delle parti e, quindi, l'equità correttiva cerca di produrre più uguaglianza", (Chamié, 2018, p.217). E' chiaro che la mancanza di cooperazione può generare abusi, sfruttamenti o approfittamenti ingiusti ed è, pertanto, fondamentale risaltare la funzione dell' “equità per cercare di ristabilire l'equilibrio rotto, ripristinare l'equivalenza (aequalitas), applicare un rimedio equo che generi uguaglianza" (Chamié, 2018, p.218).

Emblematica è la condotta tenuta nella pandemia Covid19. A causa dell'urgente necessità di maschere e guanti, alcune imprese hanno fissato un prezzo superiore a quello ragionevole. Tale condotta potrebbe costituire un abuso del diritto. In Italia, nell'applicazione della figura un momento decisivo è costituito dal caso Renault. La Corte di Cassazione argomentando alla luce dei principi costituzionali - funzione sociale ex art. 42 Cost. - e della stessa qualificazione dei diritti soggettivi assoluti, ha individuato nella buona fede "un canone generale cui ancorare la condotta delle parti, anche di un rapporto privatistico e l'interpretazione dell'atto giuridico di 
autonomia privata e, prospettando l'abuso, la necessità di una correlazione tra i poteri conferiti e lo scopo per i quali essi sono conferiti. In questo caso il superamento dei limiti interni o di alcuni limiti esterni del diritto ne determinerà il suo abusivo esercizio" (Cassazione civile, sez. III, 18 settembre 2009, n. 20106) Pertanto anche l'esercizio del recesso ad nutum previsto nel contratto può dar luogo al risarcimento del danni se non conforme alla buona fede e alla correttezza.

Nell' ipotesi del Covid 19, le imprese erano libere di determinare il prezzo di mascherine e guanti, ma non di sfruttare in modo opportunistico e irragionevole il momento, anche alla luce della particolare fragilità economica e sociale. Infatti, come affermato in una decisione recente ricorre un abuso "quando il titolare di un diritto soggettivo lo eserciti in maniera irrispettosa del principio della buona fede, cagionando uno sproporzionato sacrificio alla controparte ed al fine di conseguire risultati ulteriori rispetto a quello per cui ha il diritto è stato attribuito" (Trib. Milano, 4.03.2020, n. 2004).

Analogamente, muovendo dalla ratio degli art. 1432 e 1467 c.c.it., nelle ipotesi nelle quali le condizioni sono radicalmente mutate a causa della pandemia, se una parte offre una rimodulazione ragionevole del contratto per fronteggiare il momento alla luce delle circostanze concrete, degli interessi di entrambi i contraenti e conforme al significato economico dell'accordo e l'altra non accetta senza opporre un rilevante interesse o giustificato motivo, può essere chiamata a corrispondere un indennizzo liquidato con i criteri tratti dal 1328, comma 1, c.c. oppure le è precluso domandare l'adempimento. Trattandosi della concretizzazione della clausola di buona fede, è necessaria, come sopra, la valutazione del caso specifico e della natura ingiustificata e abusiva del rifiuto.

Ciò non è sempre facile perché spesso la realtà mostra imprese o individui costretti a trovare modi di sopravvivere in un contesto inaspettato. Si consideri un contratto di locazione commerciale di un negozio che rimane chiuso per due o tre mesi: a fronte della difficoltà di corrispondere il canone, vi potrebbe essere una persona che ne ha bisogno come fonte di sussistenza 
principale. Oppure il pagamento di una somma inferiore per le forniture, soprattutto se generale, costringe un'impresa a licenziare dipendenti o ad abbandonare linee di produzione. Una soluzione potrebbe essere, appunto, la rinegoziazione, ma non sempre porta ad un risultato effettivo. Qualora non sia avviata o fallisca, ci si può interrogare sulla opportunità di una modifica da parte del giudice. Questo potrebbe avvenire quando la differenza di posizioni fra i contraenti durante la rinegoziazione non era lontana, le parti avevano indicato nel contratto parametri di riferimento utilizzabili per la rinegoziazione, oppure - ove possibile - si possano adottare gli indici del mercato, se non sono tali da stravolgere il senso economico del contratto.

Si nota come il principio dell'abuso sia nato proprio per rispondere alla disumanizzazione del rapporto giuridico e, pur nella consapevolezza della illusorietà dell'idea che basti a moralizzare il diritto, si è rivelato uno strumento utile per rispondere all'arbitrio, all'anormalità e all'offesa al comune sentimento (Rescigno, 1965, p. 289). Tuttavia, non bisogna ritenerlo "fondato di per sé, indipendentemente da come è ricostruito e utilizzato. Tanto meno...può essere considerato un buon argomento in ragione della stigmatizzazione del comportamento da esso operata" (Velluzzi, 2019, p. 340). Sono necessarie, pertanto, sia attenzione alla motivazione e alla giustificazione del suo impiego, tentando di equilibrare le esigenze di certezza e di giustizia.

Ciò dimostra la rilevanza di un dibattito in merito al riorientamento del contratto verso i principi di solidarietà e buona fede che permetta anche di affrontare eventi che hanno indebolito significativamente economie piccole e fragili, come quella dell'Ecuador. 


\section{CONCLUSIONE}

L'ordinamento giuridico ecuadoriano stabilisce il processo di costituzione, esecuzione e risoluzione di un contratto e allo stesso modo in caso di inadempimento contrattuale, generalmente determina un risarcimento completo per il creditore interessato che dipende da fattori come il tempo e la sua gravità. Non sono stati esplorati altri rimedi che si concentrino sul mantenimento della relazione.

La solidarietà contrattuale è una dottrina fondata sui principi di equilibrio e correttezza, che cerca di prevenire gli abusi delle parti e temperare, se correttamente impiegata, i rischi derivanti da una autonomia contrattuale assoluta. Il sistema giuridico ecuadoriano a livello costituzionale sembra permeabile alla introduzione della solidarietà contrattuale. Allo stesso modo, una sua limitata applicazione può essere rinvenuta nelle decisioni della Corte Costituzionale e della Corte Nacional, le quali sembrano sviluppare un sistema giurisprudenziale ibrido simile a quello americano, influenzato fortemente però dalle radici romano-germaniche.

Si osserva, però, che in America Latina, specialmente in Ecuador, le parti tendono a diffidare l'una dell'altra, cercando di sfruttare ogni possibilità di vantaggio. Ciò apparirebbe in contrasto con una visione basata sulla cooperazione. Tuttavia, questa tendenza appare essere una condizione della modernità che sta caratterizzando tutti gli Stati: si afferma una individualismo esasperato mentre "l'appartenenza comunitaria diviene fragile, temporanea e assolutamente volontaristica, perché viene meno il collante, l'idem sentire che la genera, la legittima e l'alimenta...(si) parla di <<comunità transeunti, che in un attimo si cementano e in un altro si dissolvono>>" (Di Gregorio, L., 2019, p. 74).

Non poca influenza riveste anche il fattore economico, seppure in misura minore. Il diritto comparato e la teoria dei trapianti giuridici hanno mostrato da tempo come per la corretta ed efficace introduzione di nuovi istituti, non è sufficiente esaminare il contesto giuridico, ma è necessario considerare le condizioni sociali, economiche, politiche. Questo aspetto è ancor più importante quando si tratta di una figura 
come la solidarietà che è influenzata dal momento storico, dal luogo, dall'ideologia, dalla stessa situazione concreta. Pertanto l'ordinamento ecuadoriano, a livello solo giuridico, consente la possibilità di avvicinarsi al principio di solidarietà contrattuale, ma non è certa l' efficacia della figura. Sarà il tempo a dimostrare le modalità con cui dovrà essere applicata, le sue potenzialità e criticità. Si tratta di processi graduali come dimostra l'esperienza italiana che da una concezione rigida della buona fede si è aperta ad un uso progressivamente maggiore, riconfigurandola in ottica solidarista.

Tuttavia, in questo momento di crisi è necessario rifuggire da una concezione retorica della solidarietà e fondarla sull' "uomo concreto, con le debolezze i limiti le incapacità che ne condizionano l'esistenza e l'agire; sulla insistenza a riconoscerne l'autonomia e ad impedire e rimuovere qualsiasi attitudine o tentazione di farne l'oggetto o lo strumento dell'altrui attività; sulla rivendicazione della unicità ed irripetibilità di ciascun individuo, al di là di ogni risorsa tecnica e di qualsiasi scoperta scientifica che renda più agevole l'interrogarsi sul mistero della vita" (Rescigno, 2004, p. 398). 


\section{BIBLIOGRAFIA:}

Accatino, D. (2015). Revista chilena de derecho. La "Teoría Clásica" del Contrato y la Discusión sobre su Adaptación judicial. Vol.42 (2). Recuperado de: https://scielo. conicyt.cl/scielo.php?script=sci_arttext\&pid=S071834372015000100003\#n06

Asamblea Nacional del Ecuador. Código Civil. [CODIFICACION 2005 - 010]. (10 de mayo de 2005)

Benítez, J. (2013). Solidaridad contractual: Noción posmoderna del contrato. México DF, México: UBIJUS. Recuperado de: https://books.google. com.ec /books?id=pQioBQAAQBAJ\&pg=PA2\&lpg= PA2\&dq=noci\%C3\%B3n+posmoderna +de+la+solida ridad $\&$ source $=$ bl\&ots $=c 4 D K-9 R g R X \& \operatorname{sig}=A C f U 3 U 0$ q x 2 e K Y 47 H K x U Q h F G 9 A N B N s P o t A \&hl=es\&sa $=$ X\&ved=2ahUKEwjm1L3hvunpAhWHhOA K H W 0 U C p 4 Q 6 A E w E H o E C A s Q A Q $\# \mathrm{v}=$ onepage $\& \mathrm{q}=$ noci\%C3\%B3n\%20posmoderna $\% 20$ de\%20la\%20solidaridad $\& \mathrm{f}=$ false

Bernal-Fandiño, M. (2007). El Solidarismo Contractual-Especial Referencia al Derecho Francés-. Universitas Bogotá. $\mathrm{N}^{\circ}$ 114, pp. 17. Recuperado de: https://revistas.javeriana. edu.co/index.php/vnijuri/article/view/14588

Bernal-Fandiño, M. (2016). El principio de solidaridad como límite a la autonomía privada. Revista Jurídicas, No 13 (2), pp. 60-70. DOI: 10.17151/jurid.2016.13.2.5

Benedetti, A.M., Natoli, R.. (2020). Coronavirus, emergenza sanitaria e diritto dei contratti: spunti per un dibattito. dirittobancario.it, 20 marzo 2020, Recuperado de https://www.dirittobancario.it/editoriali/albertomaria-benedetti-e-roberto-natoli/coronavirusemergenza-sanitaria-e-diritto-dei-contratti-spunti-undibattito 
Brantt, G. (2009). La Exigencia de exterioridad en el caso fortuito: su construcción a partir de la distribución de los riesgos del contrato. Revista de Derecho de la Pontificia Universidad Católica de Valparaíso. No.33, pp.39-102. Recuperado de: https://scielo.conicyt.cl/scielo.php?script=sci_ arttext\&pid=S0718-68512009000200001

Castro, J. (2015). Caso Fortuito o Fuerza Mayor Como Causal de Término de la Relación Laboral, Requisitos del Caso Fortuito ¿̇Se confunde con la imputabilidad del empleador? Revista Chilena de Derecho del Trabajo y de la Seguridad Social. Vol.6, pp.13-35.

Castronovo, C. (1987). L'avventura delle clausole generali. Il principio di buona fede. Milano ì, Giuffrè, pp. 19-29.

Castro Ruiz, M. (2015). Como Afrontar lo Inesperado. La fuerza mayor en la contratación internacional: ¿principio o cláusula? Revista de la Facultad de Derecho de la Pontificia Universidad Católica del Perú. Vol.72, pp.441-484.

Constitución de la República del Ecuador [Const.]. (2008). (Art.136). 2da ED. CEP.

Chamié, J. (2008). Equilibrio Contractual y Cooperación entre las partes: El Deber de Revisión del Contrato. Revista de Derecho Privado. Vol.14. pp.113-138.

Chamié, J. (2018). Notas sobre algunos principios generales del derecho: una reflexión a partir de principios generales y su influencia en las obligaciones en la experiencia jurídica colombiana. Revista de la Facultad de Derecho. No.80, pp.187-237. dttps://doi.org/10.18800/ derechopucp.201801.006

Corradini, D. (1970). Il criterio della buona fede e la scienza del diritto privato, Milano, Giuffrè-

Dellacasa, M. (2015). Risoluzione per inadempimento e ricorso al processo. Rivista di diritto civile., pp. 40-91. 
Di Gregorio, L. (2019). Demopatia. Soveria Mannelli, Rubettino.

Eyzaguirre, C. (2013). Expansion y Limites De La Buena Fe Objetiva - A Proposito Del "Proyecto De PrincipiosLatinoamericanos De Derecho De Los Contratos". Revista Chilena de Derecho Privado.No.21, pp.137-211. Recuperado de: https://scielo.conicyt.cl/ pdEyf/rchdp/n21/art05.pdf

Gambino, F. (2015). Il rapporto obbligatorio, Trattato di diritto civile diretto da Sacco, R., Torino, Utet.

García Agundez, J. (1983). Obligatoriedad del Contrato y Mitigación de su Eficacia. Anuario de la Facultad de Derecho de la Universidad de Extremadura.Vol.2, pp. 343-367.

Gómez, F. (2007). El incumplimiento contractual en el derecho español. Revista para el Análisis del Derecho.No.3, pp. 10-47. Recuperado de: https://dialnet.unirioja.es/ servlet/articulo? codigo $=2328884$

Grondona, M. (2004). Solidarietà e contratto: la lettura costituzionale della clausola di buona fede. Rivista trimestrale di diritto e procedura civile, pp. 727-744.

Hinestrosa, F. 2016. La tutela del acreedor frente al deudor incumplido. Revista de Derecho Privado. No. 31, pp. 5-21. DOI:https://doi.org/10.18601/01234366.n31.01

Irti, N. (2020). Riconoscersi nella parola. Bologna, Il Mulino.

Lecuyer, H. (2010). El contrato: acto de previsión. Revista de Derecho Privado. No.18, pp. 37-56.

Lipari, N. (1997). "Spirito di liberalità" e "Spirito di solidarietà". Rivista trimestrale di diritto e procedura civile, pp. $1-25$.

Mengoni L. (2011). La responsabilità contrattuale. Castronovo C., Nicolussi, A., Albanese, A. Scritti, II, Milano Giuffrè, pp.299-353. 
Monteiro, R. (2013). El interés económico de las partes y el incumplimiento contractual. Revista de Derechos Fundamentales de la Universidad de Viña del Mar. No.10, pp.85-105.

Morgestein, W. (2015). Algunos apuntes sobre la doctrina del solidarismo contractual y sus expresiones en el concepto de información regulado por el Estatuto del Consumidor colombiano - Ley 1480 de 2011. Revista de la Facultad de Derecho y Ciencias Políticas. $\mathrm{N}^{\circ} 45$, p.196. Recuperado de: http://www.scielo. org.co/scielo.php?script=sci_abstract $\&$ pid $=$ S012038862015000100009\&lng=e\&nrm=iso\&tlng=e

Oeter, S. (2018). "Drittwirkung" dei diritti fondamentali e autonomia del diritto privato Un contributo alla dimensione giuridico funzionale nel dibattito sulla Drittwirkung. Napoli, Esi, pp. 163-204.

Patiño,H.(2011). Las causales exonerativas dela responsabilidad extracontractual ¿Por qué y cómo impiden la declaratoria de responsabilidad? Aproximación a la jurisprudencia del Consejo de Estado. Revista de Derecho Privado. No.20, pp.371-398.

Rescigno, P. (1965). L'abuso del diritto. Rivista di diritto civile, I, pp.205-290.

Rescigno, P. (2004). Laurea ad honorem conferita dalla facoltà giuridica "La Sapienza" di Roma a Sua Santità Giovanni Paolo II. Laudatio di un giurista laico., Rivista di diritto civile, I, pp. 395-399.

Rescigno, P. (2006). Solidarietà e libertà. Napoli, Jovene Editore.

-Tito, J. (2019). El ocaso del contrato: Un estudio desde la doctrina contractual clásica. Problemas en torno a su eficacia. Universitas revista de la Pontificia Universidad Javeriana. No.139. Recuperado de: https://revistas. javeriana.edu.co/files-articulos/VJ/139\%20(2019II) $/ 82562148012 /$. 
Trimarchi, P. (2008). Incentivi e rischio nella responsabilità contrattuale, Rivista di diritto civile, I, pp.341-359.

Velluzzi, V. (2019). Dietro la maschera. Abuso del diritto soggettivo e interpretazione. Ballarani, A. (ed). Novecento del diritto. Torino, Giappichelli, 315-340.

Vidal, A. (2009). Revista Chilena de Derecho Privado. La noción de incumplimiento en el "código civil". Vol.16, pp.221-258 .Recuperado de : https://scielo.conicyt.cl/scielo.php?script $=$ sci arttext\&pid=S $0718-68512009000100006$ -https://servicios.contraloria.gob.ec/ CGE_DigitalizaAcuerdosUsuarios_WEB/ WFDigitalAcuerdoResponsabilidad.aspx

Ricevuto: $16 / 06 / 2020$

Approvato: 24/07/2020

\section{Felipe Ignacio Zurita Burbano}

Ricercatore giuridico indipendente

Email: felipe.zurita.23@gmail.com

Cittá: Quito

Paese: Ecuador

\section{Francesca Benatti}

Professoressa ass. di diritto comparato Università degli Studi di Padova

Email: francesca.benatti@unipd.it

Cittá: Milano

Paese: Italia 
